[0212-7199 (2008) 25: 5; pp 237-240] ANALES DE MEDICINA INTERNA Copyright (C) 2008 ARAN EDICIONES, S.L.

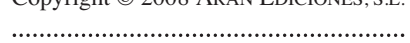

AN. MED. InTERna (Madrid) Vol. 25, N. $^{\circ} 5$, pp. $237-240,2008$

\title{
Ajo y riesgo cardiovascular
}

\author{
D. A. DE LUIS, R. ALLER \\ Sección de Endocrinologia y Nutrición Clínica. Unidad de Apoyo a la Investigacion. \\ Hospital Universitario del Río Hortega. Instituto de Endocrinologia y Nutrición Clínica. \\ Facultad de Medicina de Valladolid
}

\author{
GARLIC AND CARDIOVASCULAR RISK
}

\begin{abstract}
RESUMEN
El ajo es una de las plantas curativas más antiguas de la que tenemos referencia. En el presente trabajo, revisaremos las evidencias existentes sobre el consumo de ajo y el riesgo cardiovascular, analizando su papel sobre el perfil lipídico, el endotelio vascular y la agregación plaquetaria. El consumo regular de $5 \mathrm{~g}$ de ajo crudo dos veces al día durante 42 días disminuye los niveles de colesterol total y triglicéridos, no obstante la mayor parte de los estudios realizados en el campo de os lípidos han sido llevados a cabo en animales. En modelos animales (perros y ratas) se ha demostrado los efectos hipotensores del ajo de manera dosis dependiente. Algunos trabajos han demostrado que el ajo activa la fibrinolisis, además de suprimir el sistema de coagulación previniendo así la formación de trombos. Estos resultados se han corroborado en otro trabajo, en el cual se demuestra que el extracto alcohólico de ajo es un potente inhibidor de la agregación plaquetaria.

En resumen, los efectos del ajo sobre la disminución del riesgo cardiovascular son importantes. No obstante los estudios en humanos son escasos y todavía falta por demostrar que estos efectos disminuyan los eventos cardiovasculares así como la cantidad necesaria de ajo para obtener estos beneficios.
\end{abstract}

PALABRAS CLAVE: Ajo. Riesgo cardiovascular.

\begin{abstract}
Garlic is one of the oldest dietary vegetables. In this article, we'll review the evidences of cardiovascular risk factors and garlic consumption, analizing the influence on lipid levels, vascular endothelium and platelet aggregation. Regular consumption of $5 \mathrm{~g}$ (raw garlic) twice per day during 42 days decreases levels of cholesterol and triglyceride. However, almost all lipid studies have been realized in animals. In animal models (dogs and rats), it has been demonstrated a dose depending hypotensive effect, too. Garlic consumption has demonstrated an activation of fibrinolisis and a downregulation of coagulation, with a decrease in the rate of thrombogenesis. These results have been repeated by other work, in which a garlic alcoholic extract is a potent inhibitor of platelet aggregation.

In summary, the effects of garlic on cardiovascular risk are important. However, it is necessary to demonstrate a decrease on cardiovascular events and to elucidate the amount of garlic to obtain benefict effects, most studies are necessary.
\end{abstract}

KEY WORDS: Cardiovascular risk. Garlic.

de Luis DA, Aller R. Ajo y riesgo cardiovascular. An Med Interna (Madrid) 2008; 25: 237-240.

\section{INTRODUCCIÓN}

El ajo Allium sativum, perteneciente a la familia botánica de las liliáceas forma parte del género Allium, al que pertenecen la cebolla, los puerros o ciboulette. Su olor, penetrante y persistente, constituye su característica más notable. Esta planta es una hierba perenne resistente de 20 a 40 centímetros de altura, hojas alargadas, y flores blancas o rosadas, presenta un bulbo o "cabeza", compuesto por bulbillos o "dientes", dispuestos alrededor del tallo y envueltos en una membrana suave y sedosa.

El ajo es el más picante de todos los Allium y ha sido ampliamente utilizado en muchas culturas para finalidades medicinales y culinarias, estando ampliamente introducido en nuestra dieta mediterránea. El ajo es una de las plantas curativas más antiguas de la que tenemos referencia, ha sido cultivado por los seres humanos como planta de alimento desde hace más de 10.000 años. El ajo nace en el antiguo Turkestán (límite entre China y Afganistán e Irán) desde donde es exportado a China, India, norte de Europa y las márgenes del Mediterráneo. El Codex Ebres, un papiro médico que data del 1550 a. C. contiene 22 menciones sobre el ajo y su aplicación en el control de cardiopatía, mordeduras parásitos intestinales y tumores.

Los principales componentes activos del ajo son los amino-

Trabajo aceptado: 15 de enero de 2008 
ácidos (ácido glutamínico, arginina, ácido aspártico, leucina, lisina, valina), minerales (principalmente: manganeso, potasio, calcio y fósforo, en cantidades menores: magnesio, selenio, sodio, hierro, zinc y cobre), vitaminas (principalmente: vitamina B6, también vitamina $\mathrm{C}$ y, en cantidades menores: ácido fólico, pantoténico y niacina), aceite esencial con muchos componentes sulfurosos, aliína que, mediante la enzima alinasa, se convierte en alicina, ajoeno, producido por condensación de la alicina, quercetina, así como azucares (fructosa y glucosa).

En estos últimos años, diferentes estudios llevados a cabo han aportado un gran número de evidencias científicas que pueden justificar su uso como agente antihipertensivo, antifúngico, antimicrobiano, antitrombótico, hipoglicemiante y hipolipemiante.

En el presente trabajo, revisaremos las evidencias existentes sobre el consumo de ajo y el riesgo cardiovascular, analizando su papel sobre el perfil lipídico, el endotelio vascular y la agregación plaquetaria.

\section{AJO Y PERFIL LIPÍDICO}

En modelos animales se ha demostrado los beneficios del ajo sobre el perfil lipídico y progresión de la placa de ateroma. Uno de los primeros trabajos realizados por Efedy y cols. (1), en arterias carótidas de conejos demostró que el extracto de ajo reduce los niveles séricos de colesterol teniendo un efecto protector contra la arteriosclerosis inhibiendo el desarrollo de las lesiones en la íntima de la arteria. Otro trabajo (2) en conejos demuestra que la suplementación de ajo disminuye el colesterol y reduce la síntesis, maduración y acumulación de colágeno en las arterias. Los estudios en este campo son múltiples, consiguiendo demostrar en modelos animales; prevención de las alteraciones sobre el endotelio vascular (3), reducción de la evolución del volumen de la placa de ateroma (4) y reducción de lípidos contenidos en células arteriales (5).

También se han realizado estudios en humanos, por ejemplo en Canadá se evaluó la influencia sobre el perfil lipídico del ajo y el aceite de pescado, por separado y combinados, sobre los lípidos plasmáticos en varones hipercolesterolémicos y se concluyó que el ajo aislado producía una disminución del colesterol total y del LDL colesterol, mientras que el aceite de pescado disminuía los triglicéridos y aumentaba el LDLcolesterol. La combinación de ambos invertía el efecto del aumento de LDL-colesterol inducido por el aceite de pescado (6). En otro trabajo, el consumo regular de $5 \mathrm{~g}$ de ajo crudo dos veces al día durante 42 días disminuyó los niveles de colesterol total y triglicéridos (7).

A pesar de que estos estudios anteriores corroboren que el ajo tiene un efecto sobre los lípidos plasmáticos, también existen estudios en los que no se ha demostrado esta relación de forma significativa. En la Universidad de Bonn (8), a 25 sujetos con hipercolesterolemia moderada, se les administró una preparación de aceite de ajo comparándolos con un grupo placebo, afirmando que este preparado no tiene influencia en las lipoproteínas plasmáticas, ni en la absorción y síntesis del colesterol. Tampoco se detectaron resultados estadísticamente significativos en un estudio holandés que investigó el efecto del ajo en polvo sobre la proteína $\mathrm{C}$ reactiva y lípidos en plasma en personas fumadoras con sobrepeso, donde no hubo evidencia antiinflamatoria ni mejora en el perfil lipídico sobre los sujetos normolipémicos (9). En diseños tipo ensayo clínico tampoco se ha conseguido demostrar diferencias estadísticamente significativas. De este modo en la Universidad de Stanford se ha estudiado el efecto de una preparación comercial de ajo sobre sujetos hipercolesterolémicos frente a placebo, sin encontrar diferencias significativas en los niveles de lípidos en plasma (10), tampoco se encontraron diferencias en otros trabajos aleatorizados frente a placebo (11-12).

\section{EFECTO SOBRE LA TENSIÓN ARTERIAL Y ENDOTELIO VASCULAR}

En modelos animales (perros y ratas) se ha demostrado en la década de los 90 los efectos hipotensores y bradicardizantes del ajo de manera dosis-dependiente (13). También se ha demostrado en perros su efecto antiarrítmico dosis-dependiente por la supresión de contracciones ventriculares precoces, así como de la incidencia de taquicardia ventricular (14). Por otra parte se ha contrastado en modelos animales los efectos diuréticos de cápsulas de ajo en polvo y de su efecto antihipertensivo, provocando, a dosis elevadas, bradicardia e inversión de la onda T en el ECG (15).

Los efectos relajantes también se han demostrado en modelos animales en el músculo cardiaco (16), mostrándose en otro trabajo (17) que la fuerza contráctil de corazón se ve disminuida, hipotetizando los autores que altas dosis de este extracto podrían tener efectos no deseables. Otros grupos defienden la hipótesis de que el ajo en ratas bloquea un factor que induce la hipertensión antagonizando in vivo el efecto inhibitorio, pero los resultados no han sido estadísticamente significativos (18).

\section{EFECTO ANTIAGREGANTE PLAQUETARIO}

Chan y cols. (19) han realizado un estudio en ratas con el aceite de ajo enriquecido en dialil trisulfidico para comprobar su efecto anticoagulante, concluyendo que este compuesto tiene la capacidad de inhibir o desactivar la formación de trombina, aumentar los factores anticoagulantes, prevenir el desarrollo de la formación de trombos y aumentar la concentración de fibrinógeno en plasma a altas dosis.

Otro estudio con ajo en polvo ha demostrado que este favorece la actividad fibrinolítica además de suprimir el sistema de coagulación previniendo así la formación de trombos (20). Estos resultados se han corroborado en otro trabajo, en el cual se demuestra que el extracto alcohólico de ajo es un potente inhibidor de la agregación plaquetaria (21).

En un ensayo clínico randomizado realizado en varones con cardiopatía isquémica ha demostrado que el consumo de extracto de ajo produce una disminución de los productos de estrés oxidativo y un aumento de la dilatación de la arteria braquial (22). En otro trabajo se ha demostrado como el consumo de extracto de ajo mejora la disfunción endotelial inducida por la hiperhomocisteinemia (23).

\section{OTROS EFECTOS BENEFICIOSOS A NIVEL CARDIOVASCULAR}

Estudios realizados en ratas durante 3 semanas demuestran que el ajo y el dialil trisulfúrico mejoran el control glucémico y la tolerancia oral a la glucosa e incrementan la secreción y la sensibilidad de la insulina (24). Un compuesto del ajo llamado S- allilcisteína sulfóxido puede poseer efecto anti- 
TABLA I

COMPOSICIÓN NUTRITIVA DEL AJO (POR 100 G DE PARTE COMESTIBLE)

\begin{tabular}{|c|c|c|c|c|c|}
\hline Energía (kcal) & 110 & Calcio (mg) & 14 & Vitamina B1 (mg) & 0,16 \\
\hline$(\mathrm{kJ})$ & 460 & Hierro (mg) & 1,5 & Vitamina B2 (mg) & 0,02 \\
\hline Proteína (g) & 5,3 & Yodo (mg) & 94 & Eq. Niacina (mg) & 1,3 \\
\hline Hidratos de C (g) & 23 & Magnesio (mg) & 25 & Vitamina B6 (mg) & 0,38 \\
\hline Fibra dietética (g) & 1,1 & Zinc $(\mathrm{mg})$ & 1 & Ácido Fólico ( $\mu \mathrm{g})$ & 5 \\
\hline Grasa total (g) & 0,3 & Selenio (mg) & 2 & Vitamina B12 ( $\mu \mathrm{g})$ & 0 \\
\hline AGS $(g)$ & 0,05 & Sodio (mg) & 19 & Vitamina C (mg) & 11 \\
\hline AGM (g) & Trazas & Potasio (mg) & 529 & Retinol $(\mu g)$ & 0 \\
\hline AGP $(g)$ & 0,15 & Fósforo (mg) & 134 & Carotenos $(\mu g)$ & Trazas \\
\hline AGP/AGS & 3 & Agua $(g)$ & 70,3 & Vit. A: Eq. Retinol $(\mu \mathrm{g})$ & Trazas \\
\hline$[\mathrm{AGP}+\mathrm{AGM}] / \mathrm{AGS}$ & 3 & Alcohol (g) & 0 & Vitamina D $(\mu g)$ & 0 \\
\hline Colesterol (mg) & 0 & & & Vitamina E (mg) & 0,01 \\
\hline
\end{tabular}

AGS: ácido graso saturado; AGM: ácido graso monoinsaturado; AGP: ácido graso polinsaturado.

diabético ya que tiene el mismo efecto que la insulina o la glibenclamida (25).

También se ha evaluado el efecto antioxidante del ajo. De este modo en un trabajo realizado en ratas, utilizando el aceite de ajo contra el daño oxidativo producido por la nicotina, comprueban que este aceite es un antioxidante efectivo ya que incrementa la resistencia sobre la peroxidación de los lípidos y refuerza la actividad de las enzimas antioxidantes (26).

\section{CONCLUSIÓN}

Los trabajos revisados muestran como el ajo posee propiedades y efectos positivos sobre los lípidos plasmáticos, hipertensión, progresión de la arteriosclerosis, antiagrega- ción plaquetaria etc. A pesar de ello, son muchos los interrogantes abiertos. Por ejemplo, no está claro si los efectos demostrados en los animales son extrapolables en su integridad a las personas, tampoco esta claro la cantidad necesaria de ajo al día que es precisa ingerir para obtener los efectos deseados, ni el tipo de preparado (en crudo, extracto en polvo, en base oleosa, etc.).

Por otra parte son necesarios nuevos estudios que evalúen si estos efectos beneficiosos del consumo de ajo se traducen en una disminución de los eventos cardiovasculares y/o mortalidad asociada, de momento solo demostrado en animales (27). Por ello nos queda un largo camino que recorrer en la evaluación científica de este alimento, siempre en el contexto de una dieta equilibrada.

\section{Bibliografía}

1. Efendy JL, Simmons DL, Campbell GR, Campbell JH. The effects of the aged garlic extract, 'Kyolic', on the development of experimental atherosclerosis. Atherosclerosis 1997; 132: 37-42.

2. Mirhadi SA, Singh S, Gupta PP. Effect of garlic supplementation to atherogenic diet on collagen biosynthesis in various tissues of rabbits. Indian Heart J 1990; 42: 99-104.

3. Slowing K, Ganado P, Sanz M, Ruiz E, Tejerina T. Study of garlic extracts and fractions on colesterol plasma levels and vascular reactivity in colesterol-fead rats. J Nutr 2001; 131: 994S-9S

4. Koscielny J, Klüssendorf D. The anthiatherosclerosis effect of Allium sativum. Atherosclerosis 1999; 144: 237-49.

5. Orekhov AN, Grünwald J. Effects of garlic on atherioesclerosis. Nutrition 1997; 13: 7-8.

6. Adler AJ, Holub BJ. Effect of garlic and fish-oil supplementation on serum lipid and lipoprotein concentrations in hypercholesterolemic men. Am J. Clin Nut 1997; 65: 445-50.

7. Mahmoodi M, Islami MR, Asadi Karam G, Khaksari M, Sahenghadam Lotfi A, Study of the effect of raw garlyc consumption on the level of lipids and other bloodd biochemical factors in hyperlipidemic individuals. Pak J Pharm 2006; 19: 295-8.

8. Berthold HK, Sudhop T, von Bergmann K. Effect of garlic oil preparation on serum lipoproteins and cholesterol metabolism: A randomized controlled trial. JAMA 1998; 179: 1900-2.

9. Van Doorn MB, Espirito Santo SM, Meijer P. Effect of garlic powder on C- reactive protein and plasma lipids in overweight and smoking subjects. Am J Clin Nutr 2006; 84: 1924-9.
10. Gardner CD, Chatterjee LM, Carlson JJ. The effect of a garlic preparation on plasma lipid levels in moderately hypercholesterolemic adults. Atherosclerosis 2001; 154: 213-20.

11. Superko HR, Krauss RM. Garlic powder, effect on plasma lipids, postpandrial lipemia, low density lipoprotein particule size, high-density lipoprotein subclass distribution and lipoprotein (a). J Am Coll Cardiol 2000; 35: 321-6.

12. Isaacsohn JL, Moser M. Garlic powder and plasma lipids and lipoproteins: A multicenter, randomizer, placebo control trial. Arch Intern Med 1998 8; 158: 1189-94.

13. Martin N, Bardisa L, Pantoja C, Roman R, Vargas M. Experimental cardiovascular depressant effects of garlic (Allium sativum). J Ethnopharmacol 1992; 37: 145-9.

14. Martin N, Bardisa L, Pantoja C, Vargas M, Quezada P, Valenzuela J. Anti-arrhythmic profile of a garlic dialysate assayed in dogs and isolated atrial preparations. J Ethnopharmacol 1994; 43: 1-8.

15. Pantoja CV, Chiang LC, Norris BC, Concha JB. Diuretic, natriuretic and hypotensive effects produced by Allium sativum (garlic) in anaesthetized dogs. J Ethnopharmacol 1991; 31: 325-31.

16. Aqel MB, Gharaibah MN, Salhab AS. Direct relaxant effects of garlic juice on smooth and cardiac muscles. J Ethnopharmacol 1991; 33: 13-9.

17. Yadav RK, Verma NS. Effects of garlic (Allium sativum) extract on the Herat rate, rhythm and force of contraction in frog: A dose-dependent study. Indian J Exp Biol 2004; 42: 628-31.

18. Pedraza-Chaverri J, Tapia E, Medina-Campos O, Granados MA, Franco 
M. Garlic prevents hypertension induced by chronic inhibition of nitric oxide synthesis. Life Sciences 1998; 62: 71-7.

19. Chan KC, Yin MC, Chao WJ. Effect of diallyl trisulfide-rich garlic oil an blood coagulation and plasma activity of anticoagulation factors in rats. Food Chem Toxicol 2007; 45: 502-7.

20. Fukao H, Yoshida H, Tazawa Y, Hada T. Antithrombotic effects of odorless garlic powder both in vitro and in vitro. Biosci Biotechnol Biochem 2007; 71: 84-90.

21. Indrajit D, Khan NS, Sooranna SR. Potent activation of nitric oxide synthase by garlic: A basis for its therapeutic applications. Curr Med Res Opin 1995; 13: 257-60.

22. Williams MJ, Sutherland WH, McCormick MP, Yerman Dj, de Jong $\mathrm{Sa}$. Aged garlic extract improves endothelial function in men with CAD. Phytother Res 2003; 19: 314-9.

23. Weiss N, Ide N, Abahji T, Nill L, Keller C, Hoffmann U. Aged garlic improves homocysteine. Induced endothelial dysfunction in macro- and micro. J Nutr 2006;136: 7505-45

24. Liu CT, Hse H, Lii CK, Chen PS, Sheen LY. Effects of garlic oil and diallyl trisulfide on glycemic control in diabetic rats. Eur J Pharmacol 2005; 516: 165-73.

25. Salazar Sotolongo Y. Allium sativum 1. un agente antitrombótico diferente" Rev. Cubana Angiol y Cir Vasc 2000; 1: 155-60

26. Helen A, Rajasree CR, Krishnakumar K, Augusti KT,Vijayammal PL. Antioxidant role of oils isolated from garlic (Allium sativum Linn) and onion (Allium cepa Linn) on nicotine-induced lipid peroxidation. Vet Hum Toxicol 1999; 41: 316-9.

27. Kim JM, chang N, Kim KW, Chun HS. Dietary S-allyl-L-cysteine reduces mortality with decreased incidence of stroke and behavioral changes in stroke-prone spontaneously hypertensive rats. Biosci Biotechnol Biochem 2006; 70: 1969-71. 